\title{
ANLIXFD/CP-102547
}

\section{Magnetic imaging of a buried SmCo layer in a spring}

\section{magnet}

\author{
J. Pollmann, G. Srajer, D. Haskel, J.C. Lang, J. Maser \\ Advanced Photon Source, Argonne National Laboratory, Argonne, IL 60439 \\ J.S. Jiang, S.D. Bader \\ Materials Sciences Division, Argonne National Laboratory, Argonne, IL 60439
}

October 23, 2000

Images of the magnetic domain structure in a $1600 \AA$ thick buried SmCo layer in a $\mathrm{Fe} /$ SmCo spring magnet were obtained using a newly developed x-ray microprobe. This probe combines circularly polarizing optics with a microfocusing Fresnel zone plate to obtain a highly polarized, small cross-section x-ray beam in the energy range between 5 and $10 \mathrm{keV}$. X-ray magnetic circular dichroism was used to provide contrast between different magnetic domains as a function of the externally applied magnetic field. The obtained images show domain walls not oriented parallel to the external field but correlated with structural features in the sample. A plausible explanation is pinning of domain walls at stacking faults in the layer. The range of external fields for which the magnetic reorientation of a particular microscopic domain occurred was much smaller than the range measured for a macroscopic hysteresis loop, indicating that the reorientation is due to growth of domains developed at local nucleation points. 


\section{DISCLAIMER}

This report was prepared as an account of work sponsored by an agency of the United States Government. Neither the United States Government nor any agency thereof, nor any of their employees, make any warranty, express or implied, or assumes any legal liability or responsibility for the accuracy, completeness, or usefulness of any information, apparatus, product, or process disclosed, or represents that its use would not infringe privately owned rights. Reference herein to any specific commercial product, process, or service by trade name, trademark, manufacturer, or otherwise does not necessarily constitute or imply its endorsement, recommendation, or favoring by the United States Government or any agency thereof. The views and opinions of authors expressed herein do not necessarily state or reflect those of the United States Government or any agency thereof. 


\section{DISCLAIMER}

Portions of this document may be illegible in electronic image products. Images are produced from the best available original document. 


\section{Introduction}

Spring magnets are nanocomposites of soft and hard magnetic materials. In these composites, the soft magnet provides a high magnetic saturation, whereas the magnetically hard material provides a high coercive field [1]. Such a combination, if mutually exchangecoupled, can be used to obtain materials with a very high magnetic energy product $(B \cdot H)$. In addition, the exchange interaction between the soft and the hard material leads to a reversible demagnetization curve for the soft layer, where the magnetic moments in the soft layer are pinned at the soft layer/hard layer interface, while the moments in the bulk of the soft layer can follow an externally applied field. Upon removal of the external field, the soft layer will return to its previous alignment relative to the hard layer, thus justifying the name 'spring magnet'. These properties make spring magnets promising candidates for applications, such as permanent magnets much stronger than those currently commercially available [2].

In order to investigate the fundamental magnetization-reversal process in spring magnets, they are typically modeled as multilayer structures [3]. These layers are usually grown epitaxially by magnetron sputtering techniques. The spatial magnetic structure of the buried layers in these multilayers, however, has not been measured up to now because most magnetic domain imaging techniques, such as magnetic force microscopy (MFM) or magneto-optical Kerr-effect, are highly surface sensitive. Photoemission electron microscopy (PEEM) may be able to penetrate capping layers but cannot be used when external magnetic fields are applied. Thus, the structure of the buried layer upon magnetization reversal in an external field could not be studied directly up to now.

In this paper, we have used a newly developed polarized x-ray microprobe [4] to obtain this information. The energy of the $\mathrm{x}$ rays ( 5 to $10 \mathrm{keV}$ ) allows us to penetrate the top layers of the structure and thus to collect information on the magnetic domain structure of the buried layer while an external field is applied. The microprobe combines circularly 
polarizing with microfocusing optics to obtain a highly polarized, small cross-section $\mathrm{x}$-ray beam.

\section{Experiment}

X-ray magnetic circular dichroism (XMCD) [5] was used in this experiment in order to obtain sensitivity to the orientation of the magnetization. XMCD refers to the dependence of the $\mathrm{x}$-ray cross section $(\sigma)$ on the magnetization $(M)$ of the sample and the degree of circular polarization $\left(P_{c}\right)$ of the incident photon. For a relative angle $\theta$ between the helicity of the photon and the orientation of the magnetic moment of the absorbing atom, the $\mathrm{x}$-ray absorption cross section takes the form:

$$
\sigma \propto \sigma_{0}+M P_{c} \cos \theta,
$$

where $\sigma_{0}$ is the normal charge absorption that does not depend on the sample magnetization. The XMCD effect only gets sufficiently large close to absorption edges, thus providing a convenient means for element-specific measurements. By measuring the absorption cross section for opposite helicities, the magnetic term can be separated from the charge scattering by taking the difference of the signals. The so-called flipping ratio, $\left(I^{+}-I^{-}\right) /\left(I^{+}+I^{-}\right)$, then is a measure for the orientation $\theta$ of the local magnetic moments relative to the photon helicity.

The experiment was performed at the 4-ID insertion device beamline of SRICAT at the Advanced Photon Source, Argonne National Laboratory. To produce a circularly polarized x-ray beam with high degree of polarization, a diamond phase retarder in Bragg transmission geometry was used [6]. This beam was subsequently focused using a Fresnel microzone plate in conjunction with an order-sorting pinhole. The focused beam was circularly polarized to better than $99 \%$. The beam size, measured by knife-edge scans, was $5.3 \times 4.8 \mu \mathrm{m}^{2}$ (horizontally $\times$ vertically). These numbers are consistent with theoretical 
calculations, taking into account the demagnification of the source and the divergence of the incoming beam. The polarized flux in the focal spot was measured to be $5 \times 10^{7}$ photons/s.

The sample studied was a $\mathrm{Fe} / \mathrm{SmCo}$ spring magnet, grown on a $\mathrm{MgO}$ substrate. The sandwich structure was $(1 \mathrm{~mm} \mathrm{MgO} \mathrm{/} 200 \AA \mathrm{Fe} / 1600 \AA \mathrm{SmCo} / 200 \AA \mathrm{Fe} / 200 \AA$ $\mathrm{Ag})$. The SmCo was nominally deposited in the 2:7-phase. Local deviations from the ideal stoichiometry create Co-rich or -poor unit cells, leading to $\mathrm{SmCo}_{5}$ - or $\mathrm{SmCo}_{3}$-phases [7]. Because of the magnetic Fe layer on top of it, the buried SmCo layer is inaccessible to existing surface-sensitive magnetic measuring techniques.

Since the sample was grown on a relatively thick substrate, we used the fluorescence yield from the sample to measure the absorption. The fluorescence from the sample is proportional to the $\mathrm{x}$-ray absorption and therefore is also sensitive to the XMCD signal [8]. Measurements were performed near the Sm L3 edge (6.716 keV), monitoring the $\mathrm{L}_{\alpha}$ fluorescence intensity. The helicity of the beam was reversed at each data point, and the flipping ratio was used as a measure of the local magnetization.

Since the XMCD signal shows a strong energy dependence near the absorption edge, it is important to find the optimum energy for the experiment. This was done by performing XMCD measurements as function of energy in the fully aligned state with unfocused beam (see figure 1). The best magnetic contrast was found to be at $6.709 \mathrm{keV}$, which was the energy then used to obtain all the domain images. Magnetic dichroism contrast images were recorded for the $\mathrm{SmCo}$ layer as a function of the externally applied magnetic field. The sample was scanned in two dimensions through the microfocused beam. A magnetic field of up to $8 \mathrm{kOe}$ was applied parallel to the axis of easy magnetization. 


\section{Results}

The normalized intensity anisotropy for opposite helicities of the incoming $\mathrm{x}$-ray beam provides information about the component of the magnetic moments along the photon wavevector. Figure 2 shows four $50 \times 50 \mu \mathrm{m}^{2}$ images obtained for different applied magnetic fields. The colors in the maps correspond to the measured anisotropy, thus giving information on the magnetic structure in the SmCo layer. Clearly, domains with different orientations of the local magnetic moments can be distinguished. Boundaries between these domains do not lie parallel to the easy axis of magnetization, but at angles between $45^{\circ}$ and $90^{\circ}$. The magnetic reversal of the domains in the SmCo layer upon increase of the applied field is also resolved, showing the growth of two domains at the expense of the oppositely oriented intermediary domain.

The direction of the domain walls can be understood from the chemical structure of the SmCo layer. The axis of easy magnetization in SmCo films is given by the $c$-axis of the $\mathrm{Sm}_{2} \mathrm{Co}_{7}$ unit cell. Stacking disorders induced by the $\mathrm{SmCo}_{5}-$ or $\mathrm{SmCo}_{3}$-phases mentioned earlier will be oriented perpendicular to the easy axis. These stacking disorders effectively pin the domain walls. Deviations from this preferred orientation of domains (lower left corner of some images in figure 2) may be due to other structural defects. This assumption is supported by the fact that the sum of the fluorescence signals, corresponding to the $\mathrm{Sm}$ concentration, shows strong correlations to the magnetic structures (see figure 3).

The range over which the microscopic reorientation occurs (specified by green bars in figure 2) is small compared to the one in the macroscopic hysteresis curve (blue diamonds in figure 2). This points to the conclusion that the observed hard layer-reorientation is due to the rapid growth of local reversed domains and that the breadth of the hard layer reversal measured by magnetometry is probably due to the dispersion of nucleation fields.

In conclusion, we were able to determine for the first time the orientation of magnetic domains in a SmCo layer buried beneath another ferromagnetic layer of Fe. More local 
and global microscopic data, magnetic as well as nonmagnetic (e.g. $\mu \mathrm{XANES}$ ), will be collected to fully understand the magnetic properties of the hard layer in these spring magnet structures.

\section{Acknowledgment}

Research at the Advanced Photon Source is supported by U.S. DOE-BES under contract No. W-31-109-ENG-38. 


\section{References}

[1] E.F. Kneller and R. Hawig, IEEE Trans. Mag. 27, 3588 (1991).

[2] J.S. Jiang, E.F. Fullerton, C.H. Sowers, A. Inomata, S.D. Bader, A.J. Shapiro, R.D. Shull, V.S. Gornakov, and V.I. Nikitenko, IEEE Trans. Mag. 35, 3229 (1999).

[3] E.F. Fullerton, J.S. Jiang, M. Grimsditch, C.H. Sowers, and S.D. Bader, Phys. Rev. B 58, 12193 (1998).

[4] J. Pollmann, G. Srajer, J. Maser, J.C. Lang, C.S. Nelson, C.T. Venkataraman, and E.D. Isaacs, Rev. Sci. Instrum. 71, 2386 (2000).

[5] G. Schütz, W. Wagner, W. Wilhelm, P. Kienle, R. Zeller, R. Frahm, and G. Materlik, Phys. Rev. Lett. 58, 737 (1987).

[6] J.C. Lang and G. Srajer, Rev. Sci. Instrum. 66, 1540 (1995).

[7] M. Benaissa, K.M. Krishnan, E.F. Fullerton, and J.S. Jiang, IEEE Trans. Mag. 34, 1204 (1998).

[8] P. Strange, P.J. Durham, and B.L. Gyorffy, Phys. Rev. Lett. 67, 3590 (1991). 
Figure 1: Measured flipping ratio as a function of energy, showing the resonant enhancement close to the $\mathrm{Sm} \mathrm{L} 3$ edge. The maximum normalized magnetic contrast, located at $6.709 \mathrm{keV}$, is about $1 \%$. The dashed line serves as guide to the eye.

Figure 2: Images of magnetic domains in a buried SmCo-layer as function of the applied magnetic field. The size of the scanned area is approximately $50 \times 50 \mu \mathrm{m}^{2}$. The color of each spot corresponds to the measured flipping ratio, ranging from $-1.5 \%$ (dark blue) to $+1.5 \%$ (red). The easy axis of magnetization lies parallel to the vertical axis of the images. The sample first was fully aligned in one direction by applying a field of $+7.8 \mathrm{kOe}$ in direction of the easy axis. Then the reorientation of domains was observed while applying a field in the opposite direction. The applied fields were $-6.22 \mathrm{kOe}(\mathrm{a})$, $-6.24 \mathrm{kOe}(\mathrm{b}),-6.28 \mathrm{kOe}(\mathrm{c})$, and $-6.30 \mathrm{kOe}(\mathrm{d})$. Blue diamonds show a branch of the macroscopic hysteresis of the sample.

Figure 3: Magnetic (a) and charge (b) images for an applied magnetic field of $-6.28 \mathrm{kOe}$. The signal was obtained from the difference (a) and the sum (b), respectively, of the fluorescence signal measured for opposite helicities of the incoming x-ray beam. The pixel size was chosen to oversample the area twice in each direction. 

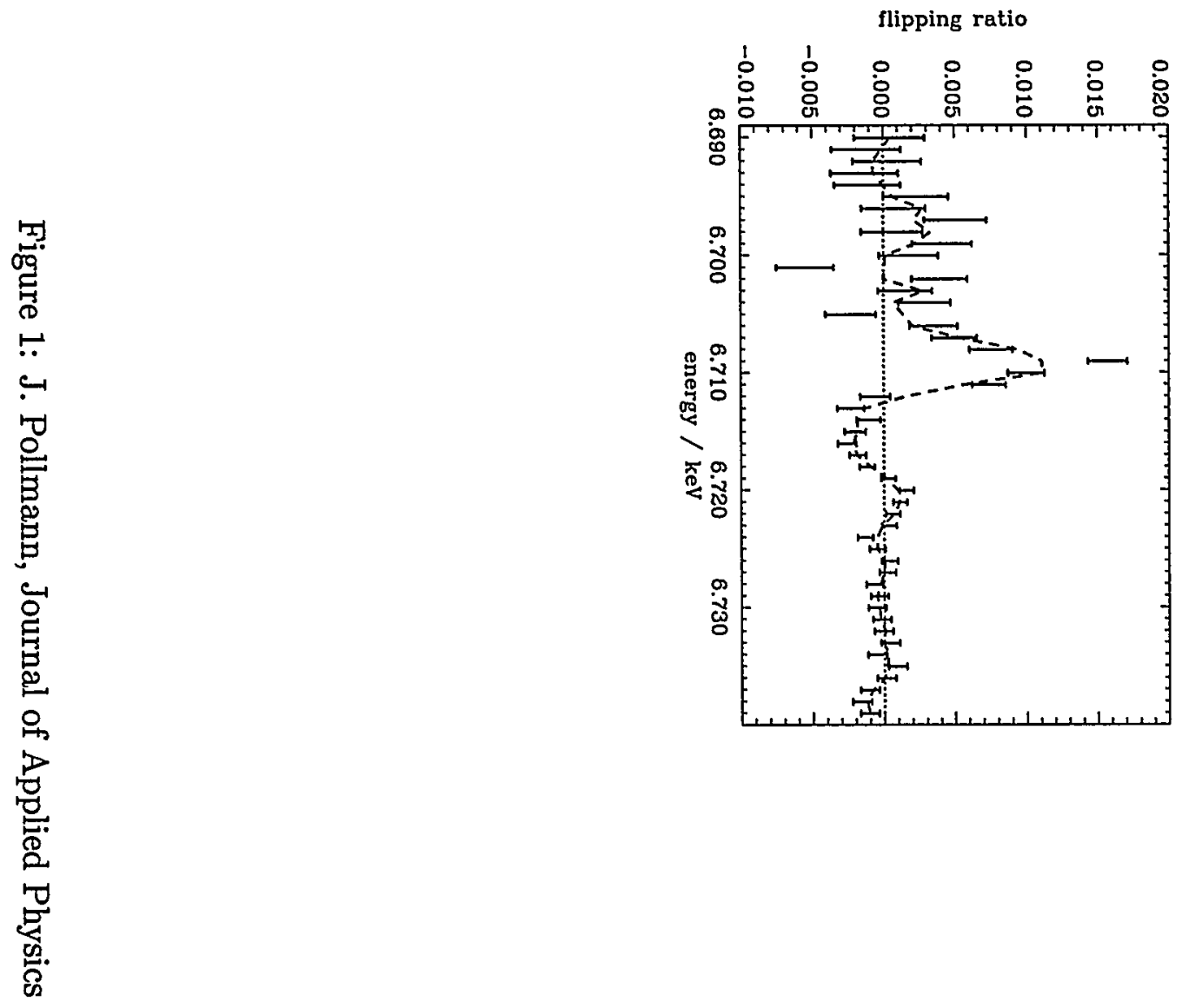


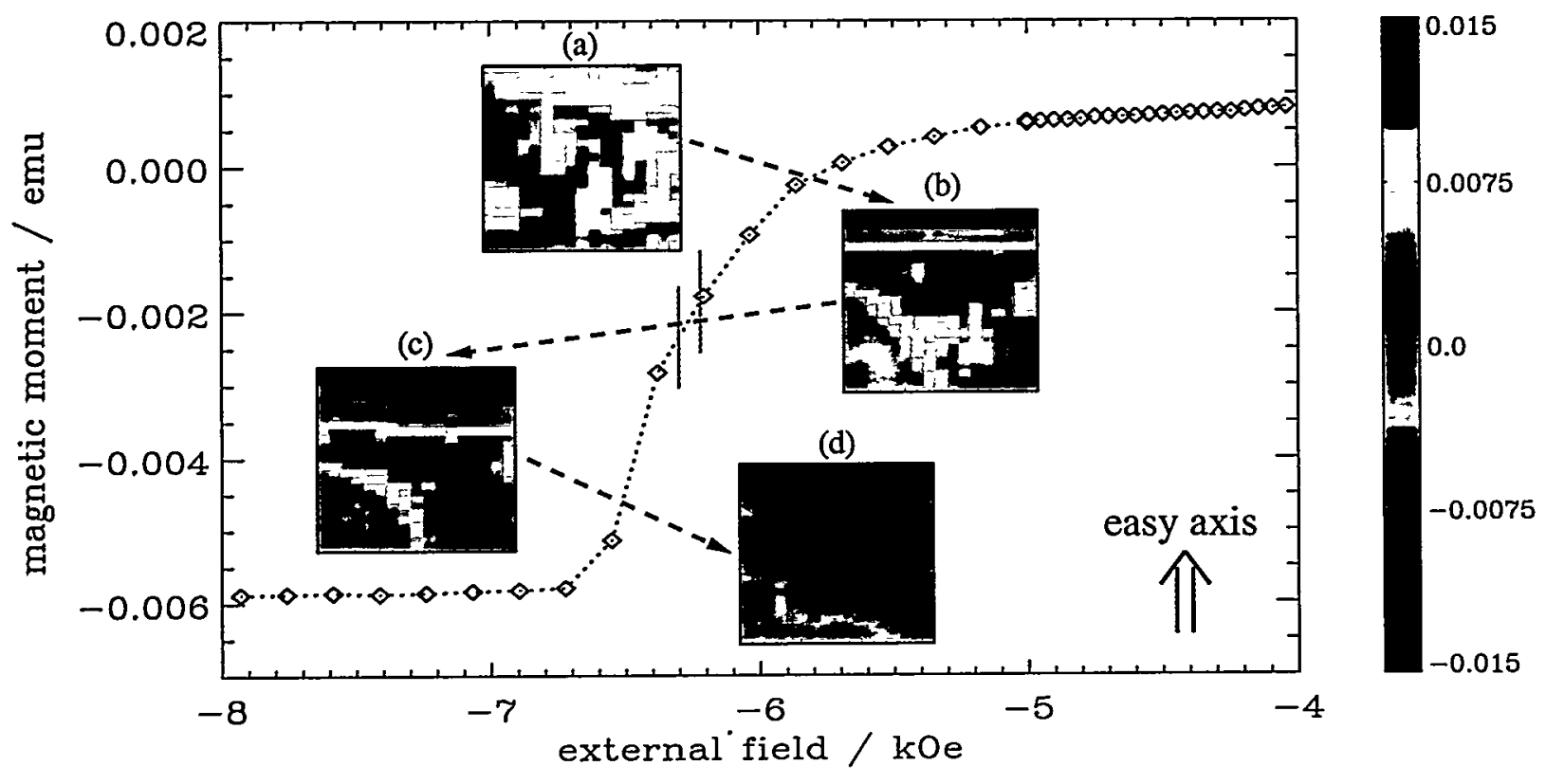

Figure 2: J. Pollmann, Journal of Applied Physics 
(a)

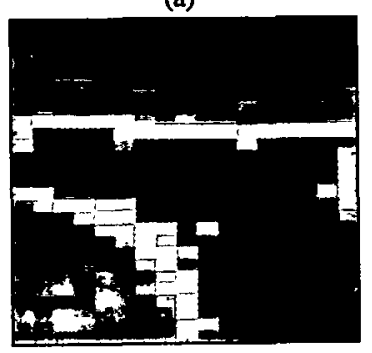

(b)

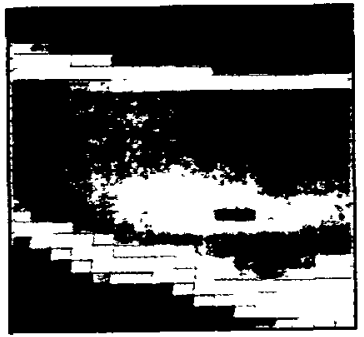

Figure 3: J. Pollmann, Journal of Applied Physics 